\title{
Inhibition of nasopharyngeal carcinoma cell proliferation and synergism of cisplatin with silvestrol and episilvestrol isolated from Aglaia stellatopilosa
}

\author{
MAELINDA DAKER ${ }^{1}$, JIUN-TZEN YEO ${ }^{2}$, NORHASIMAH BAKAR ${ }^{1}$, \\ ASMA' SAIYIDATINA AISHAH ABDUL RAHMAN ${ }^{2}$, MUNIRAH AHMAD $^{1}$, \\ TIONG-CHIA YEO ${ }^{2}$ and ALAN SOO-BENG $\mathrm{KHOO}^{1}$ \\ ${ }^{1}$ Molecular Pathology Unit, Cancer Research Centre, Institute for Medical Research, Kuala Lumpur 50588; \\ ${ }^{2}$ Sarawak Biodiversity Centre, KM20, Semengoh, Kuching, Sarawak 93990, Malaysia
}

Received February 4, 2015; Accepted December 1, 2015

DOI: 10.3892/etm.2016.3201

\begin{abstract}
Nasopharyngeal carcinoma (NPC) is a type of tumour that arises from the epithelial cells that line the surface of the nasopharynx. NPC is treated with radiotherapy and cytotoxic chemotherapeutic drugs such as cisplatin and 5-fluorouracil. However, current strategies are often associated with potential toxicities. This has prompted efforts to identify alternative methods of treatment. The present study aimed to investigate silvestrol and episilvestrol-mediated inhibition of cell proliferation in human NPC cells. The growth kinetics of NPC cells treated with silvestrol or episilvestrol were monitored dynamically using a real-time, impedance-based cell analyzer, and dose-response profiles were generated using a colorimetric cell viability assay. Furthermore, apoptosis was evaluated using flow cytometry and high content analysis. In addition, flow cytometry was performed to determine cell cycle distribution. Finally, the effects of combining silvestrol or episilvestrol with cisplatin on NPC cells was examined. Apoptosis was not observed in silvestrol and episilvestrol-treated NPC cells, although cell cycle perturbation was evident. Treatment with both compounds induced a significant increase in the percentage of cells in the $\mathrm{G}_{2} / \mathrm{M}$ phase, as compared with the control. In vitro cultures combining silvestrol or episilvestrol with cisplatin showed synergistic effects against NPC cells. The results of the present study suggested that silvestrol and episilvestrol had an anti-tumour activity in NPC cells. Silvestrol and episilvestrol, particularly in combination with cisplatin, merit further investigation, so as to determine the
\end{abstract}

Correspondence to: Mrs. Maelinda Daker, Molecular Pathology Unit, Cancer Research Centre, Institute for Medical Research, Jalan Pahang, Kuala Lumpur 50588, Malaysia

E-mail: mae_cinnamon@yahoo.com

Key words: Aglaia stellatopilosa, cell cycle arrest, cell proliferation, cisplatin, episilvestrol, nasopharyngeal carcinoma, silvestrol, synergism cellular mechanisms underlying their action(s) as anti-NPC agents.

\section{Introduction}

Nasopharyngeal carcinoma (NPC) is a type of cancer that affects the epithelial cells of the nasopharynx (1). Although NPC has a low prevalence among Caucasian populations, the disease has an exceptionally high incidence rate in the Eastern Malaysian state of Sarawak, particularly in the Bidayuh ethnic community (2). NPC is also prevalent among populations in Southeast Asia and Southern China, as well as Inuit populations in Alaska and certain ethnic groups in North Africa $(1,3)$. Concurrent chemoradiation is the current standard therapy for NPC, although this method appears to be more effective in patients with early stage NPC, as compared with patients with advanced stage NPC and distant tumour metastasis $(3,4)$. One of the treatment strategies being investigated for NPC involves the addition of another therapeutic agent to the combination of cisplatin and 5-fluorouracil, the standard chemotherapeutic drugs for the treatment of NPC (4). This approach requires a novel target agent that functions synergistically with the standard chemotherapy drugs to treat NPC.

The use of plant components as therapeutic agents has attracted much attention. Higher plants, specifically plants used in traditional medicine or as dietary supplements, are the source of a considerable number of natural product-derived drugs (5). Aglaia is a genus of plant belonging to the family Meliaceae, and can be found primarily in the forests in tropical Asia (6). Several species within the genus are known to be sources of cyclopenta[b]benzofuran flavaglines, a novel class of compound with a unique structure that has been shown to be antineoplastic (5). One member of this class of compounds, silvestrol and its 5'-epimer episilvestrol, are isolated from the twig, fruit, and bark of Aglaia stellatopilosa, a species endemic to Borneo (7). The mechanism underlying the anti-proliferative effects of the cyclopenta[b]-benzofurans occurs via inhibition of protein synthesis (8). Kinghorn et al (5) described novel plant bioactive agents with potential cancer chemotherapeutic properties, including silvestrol. Investigations into the 
phytochemical effects, synthetic methods, biological evaluation and mechanism of action of cyclopenta[b]-benzofurans are described in Pan et al (9). Rocaglates, silvestrol and episilvestrol are translation initiation inhibitors (10). However, to the best of our knowledge, the role of silvestrol and episilvestrol in the treatment of NPC has yet to be evaluated.

The aim of the present study was to evaluate the capacity of silvestrol and episilvestrol to inhibit proliferation, induce apoptosis and perturb the cell cycle in NPC cells. The results demonstrated that both silvestrol and episilvestrol are effective at inhibiting the proliferation of NPC cells in vitro by blocking the $\mathrm{G}_{2} / \mathrm{M}$ transition in the cell cycle. In addition, in combination with cisplatin, the two compounds exhibited a synergistic effect against NPC cells. These results suggested that silvestrol and episilvestrol may serve as NPC-targeting compounds in combination with existing chemoradiation treatment regimens.

\section{Materials and methods}

Chemicals. Silvestrol (Fig. 1) and episilvestrol (Fig. 2) were purchased from Cerylid Biosciences. Ltd. (Richmond, Australia).

Cell lines and culture. HK1, an Epstein-Barr virus (EBV)-negative NPC cell line (11), was provided by Professor George Tsao (Department of Anatomy, Faculty of Medicine, University of Hong Kong, Hong Kong, China). C666-1, an EBV-positive NPC cell line (12), was donated by Professor Kwok-Wai Lo (Department of Anatomical and Cellular Pathology, Faculty of Medicine, Chinese University of Hong Kong, Hong Kong, China). HK1 was maintained in the exponential growth phase in RPMI-1640 medium supplemented with $10 \%$ heat-inactivated foetal calf serum (FCS), $10 \mathrm{U} / \mathrm{ml}$ penicillin and $10 \mu \mathrm{g} / \mathrm{ml}$ streptomycin (all from Gibco; Thermo Fisher Scientific, Inc., Waltham, MA, USA) at $37^{\circ} \mathrm{C}$ in a humidified atmosphere containing $5 \% \mathrm{CO}_{2}$. C666.1 was maintained under similar conditions, although the FCS concentration was increased to $15 \%$. Passage levels of the NPC cells were in the range of 10-30. The identity of HK1 and C666.1 cells were confirmed by DNA fingerprinting using an AmpFISTR Identifiler ${ }^{\circledR}$ Polymerase Chain Reaction (PCR) Amplification kit (part no. 4322288; Applied Biosystems; Thermo Fisher Scientific, Inc.). The short tandem repeat profiles were consistent with published data (13). Detection of mycoplasma using an e-Myco ${ }^{\mathrm{TM}}$ Mycoplasma PCR Detection kit (cat. no. 25235; Intron Biotechnology, Inc., Seongnam, Korea) were conducted routinely and contamination-free cells were used throughout this study. Mycoplasma-free stocks were frozen in $10 \% \mathrm{v} / \mathrm{v}$ dimethyl sulfoxide (DMSO; Sigma-Aldrich, St. Louis, MO, USA), $40 \%$ v/v FCS and 50\% v/v RPMI-1640, then stored in liquid nitrogen for subsequent re-culturing.

Sulforhodamine $B$ (SRB) bioassay. SRB assays were conducted in order to ascertain the stability of silvestrol and episilvestrol activity against the NCI-H460 non-small cell lung cancer and MCF-7 breast cancer cell lines over a period of time. Both cell lines were obtained from American Type Culture Collection (Manassas, VA, USA), and were maintained in RPMI-1640 medium supplemented with $10 \%$ heat-inactivated $\mathrm{FCS}$ at $37^{\circ} \mathrm{C}$ in $5 \% \mathrm{CO}_{2}$. For the SRB assay, $0.1 \%$ (w/v) gentamycin (Amresco, LLC, Solon, OH, USA) was added to the culture medium, after which $100 \mu$ cells were plated in 96-well flat bottomed microtiter plates (Nalge Nunc International, Penfield, NY, USA) at 7,500 cells/well and 10,000 cells/well for NCI-H460 and MCF-7, respectively. The cells were incubated for 24 hours for recovery, after which $100 \mu 1$ culture medium or culture medium containing silvestrol or episilvestrol $(3819,381.9,38.19,3.819$ or $0.3819 \mathrm{nM})$ was added to the wells for $72 \mathrm{~h}$. Subsequently, the cells were fixed with $10 \%(\mathrm{w} / \mathrm{v})$ trichloroacetic acid (Scharlab, S.L., Barcelona, Spain) at $4^{\circ} \mathrm{C}$ for $1 \mathrm{~h}$. After five washings with reversed osmosis water, the cells were stained with $0.4 \%$ (w/v) SRB dye (MP Biomedicals, Santa Ana, CA, USA) in 1\% (v/v) acetic acid (Merck Millipore, Darmstadt, Germany) for $10 \mathrm{~min}$ at room temperature. Unbound stain was removed by washing three times with $1 \%$ acetic acid. The plates were then air-dried and the bound protein stain was solubilized with $100 \mu \mathrm{l}$ of $10 \mathrm{mM}$ Tris base (Avantor Performance Materials, Center Valley, PA, USA). The optical density was read at $515 \mathrm{~nm}$ using the Sunrise Basic Microplate Reader from Tecan Group Ltd. (Männedorf, Switzerland).

$x$ CELLigence cell proliferation assay. HK1 cells were seeded at a density of $1 \times 10^{4}$ cells/well into an E-Plate 16 (ACEA Biosciences, Inc., San Diego, CA, USA). For C666.1 cells, $3 \times 10^{4}$ cells/well were seeded. At $24 \mathrm{~h}$ following seeding, the culture medium was aspirated and replaced with fresh medium containing 6.25 or $50 \mathrm{nM}$ silvestrol or episilvestrol. The compounds were dissolved in DMSO with a final concentration of DMSO in the cell culture $\leq 1.0 \%$. Vehicle control cultures received DMSO alone. Cells treated with $33.3 \mu \mathrm{M}$ cisplatin served as the positive control. Cells were monitored dynamically for $\sim 70 \mathrm{~h}$ using the impedance-based xCELLigence real-time cell analyzer (ACEA Biosciences, Inc.). The cell index, automatically calculated from the change in electrical impedance as the living cells interacted with electrodes in the E-plate wells, correlated with the number of cells, viability and/or cytotoxicity over time.

MTS cell viability assay. A total of $1 \times 10^{4} \mathrm{HK} 1$ cells/well or $3 \times 10^{4}$ C666.1 cells/well were seeded into 96-multiwell microtiter plates using a Hamilton Microlab ${ }^{\circledR}$ STARlet robotic liquid handling workstation (Hamilton Robotics, Inc., Reno, $\mathrm{NV}$, USA). At $24 \mathrm{~h}$ following seeding, the medium was aspirated and replaced with fresh medium containing various concentrations of silvestrol or episilvestrol. Vehicle control cultures received DMSO alone. The cells were then incubated for $24 \mathrm{~h}$ at $37^{\circ} \mathrm{C}$ in an atmosphere containing $5 \% \mathrm{CO}_{2}$. The number of viable cells at the end of the incubation period was measured using a CellTiter $96{ }^{\circledR} \mathrm{AQ}_{\text {ueous }}$ One Solution Cell Proliferation (MTS) assay (Promega Corporation, Madison, WI, USA). Absorbance at $490 \mathrm{~nm}$ was read using an EnVision multilabel plate reader (PerkinElmer, Inc., Waltham, MA, USA) and subtracted with non-specific absorbance measured at $630 \mathrm{~nm}$. Wells containing medium without cells served as blanks. Cell viability was calculated as a percentage compared to the control cells, which were arbitrarily assigned $100 \%$ viability. The half maximal inhibitory concentration $\left(\mathrm{IC}_{50}\right)$ values, defined as the concentration that inhibited $50 \%$ cell 


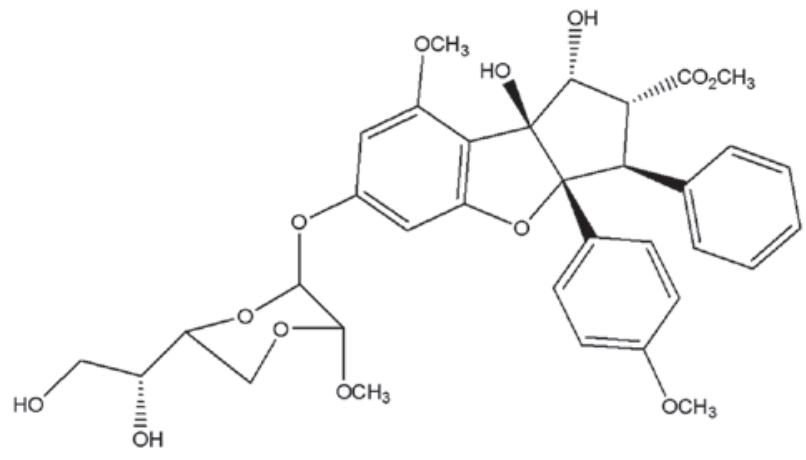

Figure 1. Chemical structure of silvestrol.

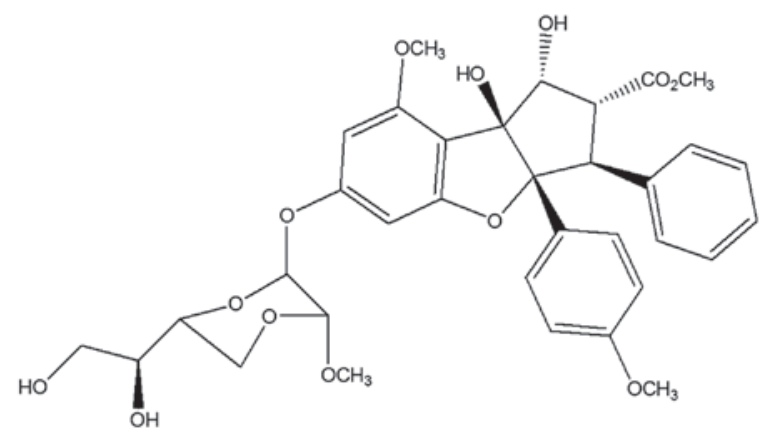

Figure 2. Chemical structure of episilvestrol.

growth relative to control cells, were graphically obtained from the dose-response curves.

Apoptosis assay. HK1 and C666.1 cells were seeded at a density of $1 \times 10^{6}$ cells $/ \mathrm{ml}$ in culture dishes containing RPMI-1640 medium supplemented with 10 (HK1) or 15\% (C666.1) heat-inactivated FCS, and allowed to adhere and reach $\sim 80 \%$ confluence overnight at $37^{\circ} \mathrm{C}$. Subsequently, the medium was aspirated and the cells were treated with $50 \mathrm{nM}$ silvestrol or episilvestrol. Vehicle control cultures received DMSO alone, whereas cells treated with $100-175 \mu \mathrm{M}$ cisplatin served as the control. Apoptosis was evaluated at $24 \mathrm{~h}$ following treatment using a FACSCalibur flow cytometry system (model no. 342975; BD Biosciences, San Jose, CA, USA) using an Annexin V-fluorescein isothiocyanate (FITC) Apoptosis Detection kit (cat. no. 556547; BD Pharmingen, San Diego, CA, USA). Data acquisition and analysis were performed using BD CellQuest Pro software, version 6.0 (BD Biosciences). A total of $1 \times 10^{4}$ events were collected for each sample. The lower right and upper right quadrants represented cells undergoing apoptosis. Annexin V-FITC-propidium iodide-stained cells were imaged using an IN Cell Analyzer 2000 (GE Healthcare Bio-Sciences, Pittsburgh, PA, USA) with a $20 \mathrm{X}$ objective. Hoechst 33342 (Cell Signaling Technology, Inc., Danvers, MA, USA) was used for nuclear staining. Briefly, $10 \mu \mathrm{l}$ Hoechst 33342 was added to the cell suspension to a final concentration of $1 \mu \mathrm{g} / \mathrm{ml}$, and then incubated in the dark for $15 \mathrm{~min}$. The following filter combinations were used: Green (490/20 ex., 525/20 em.) for detection of Annexin V-FITC; red (579/34 ex., 624/40 em.) for detection of propidium iodide; and blue (350/50 ex., 455/50 em.) for detection of Hoechst 33342.
Cell cycle analysis assay. HK1 and C666.1 cells were seeded and grown in culture dishes, as described for the apoptosis assay. Cultured cells were pulsed with $10 \mu \mathrm{M}$ bromodeoxyuridine (BrdU) daily. Cell cycle progression was determined 24 and $48 \mathrm{~h}$ following treatment on a FACSCalibur flow cytometry system using an FITC BrdU Flow kit (cat. no. 559619; BD Pharmingen). ModFit $L T^{\mathrm{TM}}$ software, version 3.3.11 from Verity Software House, Inc. (Topsham, ME, USA) was used to analyze the DNA patterns in the flow cytometry histograms.

Combined drug analysis. Cell seeding was performed as described above for the MTS cell viability assay using the Hamilton Microlab ${ }^{\circledR}$ STARlet robotic liquid handling workstation. To maintain similar experimental conditions, 96-multiwell microtiter plates were assigned simultaneously for single-drug and two-drug treatment (14). At $24 \mathrm{~h}$ following seeding, non-fixed ratio combinations of silvestrol-cisplatin or episilvestrol-cisplatin were evaluated (Table I). Following drug treatment, the microtiter plates were incubated for a further $24 \mathrm{~h}$ following which an MTS assay was conducted to determine cell viability. Drug interaction was determined using the previously described combination index (CI) method (14). CalcuSyn version 2.0 software (Premier Biosoft International, Palo Alto, CA, USA) was used to generate the dose-response curves, dose-effect analysis, and CI-effect plot. A CI $<1$ implied synergism, $\mathrm{CI}=1$ was additive, and $\mathrm{CI}>1$ implied antagonism. In addition, $\mathrm{CI}<0.1$ implied very strong synergism, $\mathrm{CI}=0.1-0.3$ implied strong synergism, $\mathrm{CI}=0.3-0.7$ implied synergism, $\mathrm{CI}=0.7-0.85$ was moderate synergism, $\mathrm{CI}=0.85-0.9$ implied slight synergism (14).

Statistical analysis. Calculations were performed using IBM ${ }^{\circledR}$ SPSS $^{\circledR}$ version 22.0 statistical software (IBM SPSS, Armonk, NY, USA). Differences between mean values were evaluated with a one-way analysis of variance and Tukey's post-hoc analysis. $\mathrm{P}<0.05$ was considered to indicate a statistically significant difference.

\section{Results}

Compound profiles. HPLC analysis of silvestrol and episilvestrol demonstrated that the compounds were pure (Figs. 3 and 4).

Stability of silvestrol and episilvestrol. Preliminary data (unpublished) from on-going experiments demonstrated that the silvestrol and episilvestrol compounds were stable over time. Silvestrol and episilvestrol reconstituted with $100 \%$ DMSO and stored at $-20^{\circ} \mathrm{C}$ produced consistent $\mathrm{GI}_{50}$ results as determined by the SRB bioassay $(72 \mathrm{~h}$ treatment period) on NCI-H460 non-small cell lung cancer and MCF-7 breast adenocarcinoma cell lines (Tables II and III).

Dynamic monitoring of cell proliferation. Based on screening experiments on NCI-H460 and MCF-7 cell lines (Tables II and III), the effective inhibition concentrations of silvestrol and episilvestrol were in the nano-molar range. Therefore, one low and one moderate dose were selected for use in the xCELLigence system; a real-time cell proliferation, viability and cytotoxicity analyzer. The cell index generated represents growth over time. 
Table I. Description of CI values for each fraction of the cells and the corresponding DRI.

A, HK1 cell line

\begin{tabular}{|c|c|c|c|c|c|c|}
\hline Compound (nM) & $\begin{array}{l}\text { Fraction } \\
\text { affected }\end{array}$ & CI & Synergism & $\mathrm{DRI}^{\mathrm{a}}$ & Cisplatin $(\mu \mathrm{M})$ & $\begin{array}{c}\text { Cisplatin } \\
\text { DRI }\end{array}$ \\
\hline \multicolumn{7}{|l|}{ Silvestrol } \\
\hline 5 & 0.211 & 0.04 & Very strong & 98.879 & 12.5 & 33.057 \\
\hline 10 & 0.208 & 0.087 & Very strong & 47.213 & 25 & 15.128 \\
\hline 50 & 0.347 & 0.016 & Very strong & 83.851 & 50 & 236.646 \\
\hline 500 & 0.391 & 0.070 & Very strong & 15.067 & 100 & 298.111 \\
\hline 1,000 & 0.336 & 0.294 & Strong & 3.603 & 150 & 62.114 \\
\hline 5,000 & 0.394 & 0.645 & Synergism & 1.567 & 200 & 158.505 \\
\hline \multicolumn{7}{|l|}{ Episilvestrol } \\
\hline 10 & 0.172 & 0.209 & Strong & 778.01 & 25 & 4.811 \\
\hline 50 & 0.270 & 0.025 & Very strong & $7.67 \times 10^{6}$ & 50 & 40.295 \\
\hline 500 & 0.324 & 0.014 & Very strong & $9.83 \times 10^{7}$ & 100 & 71.468 \\
\hline 1,000 & 0.261 & 0.093 & Very strong & $1.61 \times 10^{5}$ & 150 & 10.720 \\
\hline 5,000 & 0.440 & 0.003 & Very strong & $1.03 \times 10^{11}$ & 200 & 399.788 \\
\hline
\end{tabular}

B, C666.1 cell line

\begin{tabular}{|c|c|c|c|c|c|c|}
\hline Compound (nM) & $\begin{array}{l}\text { Fraction } \\
\text { affected }\end{array}$ & CI & Synergism & $\mathrm{DRI}^{\mathrm{a}}$ & Cisplatin $(\mu \mathrm{M})$ & $\begin{array}{c}\text { Cisplatin } \\
\text { DRI }\end{array}$ \\
\hline \multicolumn{7}{|l|}{ Silvestrol } \\
\hline 5 & 0.350 & 0.098 & Very strong & 83.104 & 33.3 & 11.638 \\
\hline 10 & 0.467 & 0.048 & Very strong & 230.427 & 66.7 & 23.053 \\
\hline 50 & 0.378 & 0.570 & Synergism & 12.633 & 266.7 & 2.037 \\
\hline 500 & 0.445 & 0.644 & Synergism & 3.297 & 400 & 2.936 \\
\hline 1,000 & 0.561 & 0.214 & Strong & 8.276 & 400 & 10.735 \\
\hline \multicolumn{7}{|l|}{ Episilvestrol } \\
\hline 5 & 0.461 & 0.033 & Very strong & 114.652 & 33.3 & 41.370 \\
\hline 10 & 0.451 & 0.071 & Very strong & 52.574 & 66.7 & 19.103 \\
\hline 50 & 0.647 & 0.038 & Very strong & 58.864 & 133.3 & 46.547 \\
\hline 500 & 0.564 & 0.444 & Synergism & 2.786 & 266.7 & 11.700 \\
\hline 1,000 & 0.670 & 0.325 & Synergism & 3.666 & 400 & 18.987 \\
\hline
\end{tabular}

${ }^{a}$ DRI values $>1$ are beneficial, and the greater the DRI values, the greater the dose reduction for a given therapeutic effect (14). CI, combination index; DRI, drug reduction index.

Proliferation of HK1 cells cultured in $6.25 \mathrm{nM}$ silvestrol was not inhibited (Fig. 5A). However, $50 \mathrm{nM}$ silvestrol exerted an immediate inhibitory effect and caused near-static cell index compared with the control cells. This observation suggests that a lower concentration of silvestrol $(6.25 \mathrm{nM})$ enhanced proliferation more than the vehicle control-treated cells, whereas a higher concentration of silvestrol $(50 \mathrm{nM})$ could inhibit cell proliferation. Similar observations were obtained with episilvestrol. A total of $50 \mathrm{nM}$ silvestrol or episilvestrol were as effective as $33.33 \mu \mathrm{M}$ cisplatin in reducing C666.1 cell index (Fig. 5B). Following treatment, there was a rapid decline in cell index. However, C666.1 cell proliferation was not entirely inhibited by $6.25 \mathrm{nM}$ silvestrol or episilvestrol, although the cell index generated was markedly lower compared with that of the control, indicating that the hyper-proliferation effect observed in HK1 cells was cell-specific. The pattern of growth inhibition by $50 \mathrm{nM}$ silvestrol and episilvestrol were comparable to that of cisplatin, a standard NPC chemotherapy drug. Since $50 \mathrm{nM}$ silvestrol and episilvestrol were sufficient for inhibiting cell proliferation, all further experiments were conducted at this minimum concentration only.

Determination of viable cells. To further examine the anti-proliferative effects exhibited by the xCELLigence system, HK1 and C666.1 cells treated with various concentrations of silvestrol (Fig. 6A) and episilvestrol (Fig. 6B) were assessed for viability using an MTS assay. In HK1 cells, increasing the treatment concentrations from $50 \mathrm{nM}$ to 10 and 
Table II. $\mathrm{GI}_{50}$ for silvestrol.

\begin{tabular}{lcc}
\hline & \multicolumn{2}{c}{$\mathrm{GI}_{50}$ value $(\mathrm{nM})$} \\
\cline { 2 - 3 } SRB bioassay performed & NCI-H460 & MCF-7 \\
\hline First test & 16.90 & 19.71 \\
3 months & 17.81 & 18.50 \\
6 months & 19.70 & 19.02 \\
9 months & 18.10 & 16.90 \\
12 months & 18.60 & 17.60 \\
\hline
\end{tabular}

$\mathrm{GI}_{50}$, concentration for $50 \%$ of maximal inhibition of cell proliferation; SRB, sulforhodamine B.

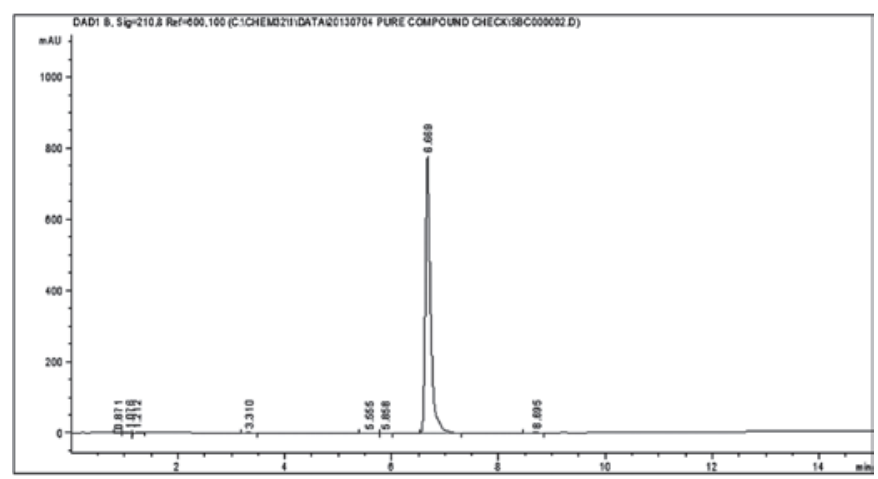

Figure 3. High-performance liquid chromatography profile of silvestrol.

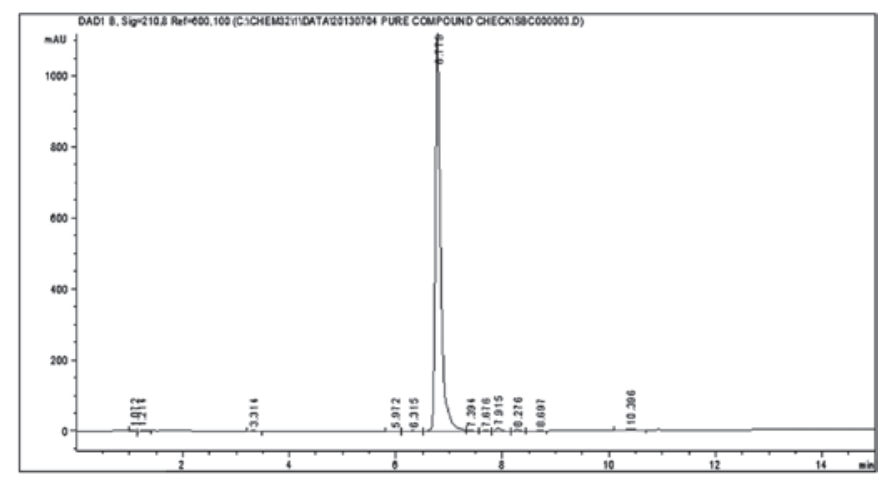

Figure 4. High-performance liquid chromatography profile of episilvestrol.

100-fold higher did not elicit further response, as evidenced by the near-plateau in cell viability $\geq 50 \mathrm{nM}$ silvestrol and $\geq 50 \mathrm{nM}$ episilvestrol. The trend in effect of silvestrol and episilvestrol against C666.1 cell proliferation was not as smooth, as compared with HK1. However, both compounds were potent against EBV-positive C666.1 NPC cells, with the effective concentrations required to inhibit $\mathrm{IC}_{50}$ values attainable within 24 h compared with EBV-negative HK1 NPC cells (Table IV). These results are concordant with those obtained from xCELLigence dynamic monitoring of cell proliferation, whereby following treatment with $50 \mathrm{nM}$ silvestrol or episilvestrol, the cell index of C666.1 cells continued to decline over time, whereas that of the HK1 cells remained static. The $\mathrm{IC}_{50}$ value of episilvestrol in C666.1 cells was markedly lower
Table III. GI $_{50}$ for episilvestrol.

\begin{tabular}{lcr}
\hline & \multicolumn{2}{c}{$\mathrm{GI}_{50}$ value $(\mathrm{nM})$} \\
\cline { 2 - 3 } SRB bioassay performed & NCI-H460 & MCF-7 \\
\hline First test & 17.96 & 19.09 \\
2 months & 15.60 & 18.70 \\
\hline GI $_{50}$, concentration for 50\% of maximal inhibition of cell prolifera- \\
tion; SRB, sulforhodamine B.
\end{tabular}

Table VI. $\mathrm{IC}_{50}$ values for the anti-proliferative activity of silvestrol and episilvestrol against NPC cells.

\begin{tabular}{lcc}
\hline & \multicolumn{2}{c}{$\mathrm{IC}_{50}$ value at $24 \mathrm{~h}(\mathrm{nM})$} \\
\cline { 2 - 3 } Cell line & Silvestrol & Episilvestrol \\
\hline C666.1 & 484 & 259 \\
HK1 & $>5,000$ & $>5,000$ \\
\hline
\end{tabular}

$\mathrm{IC}_{50}$, half maximal inhibitory concentration; NPC, Nasopharyngeal carcinoma.

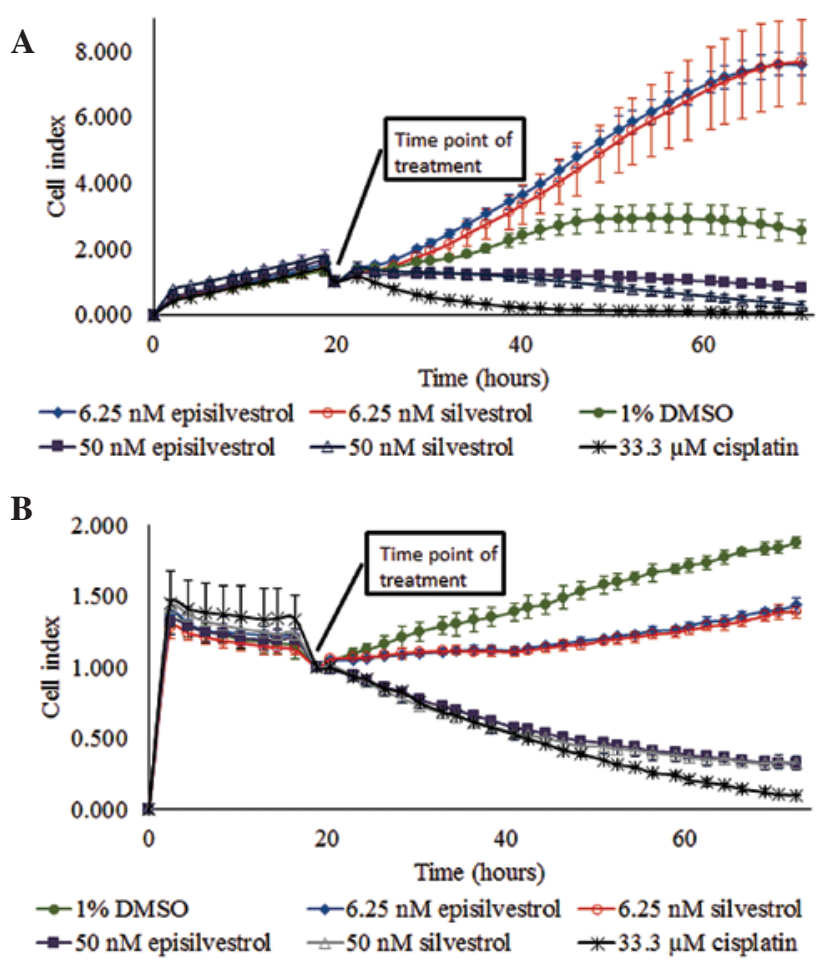

Figure 5. Real-time analysis of NPC cell proliferation with or without silvestrol or episilvestrol. The effect of various concentrations of silvestrol or episilvestrol on (A) HK1 and (B) C666.1 cell proliferation, monitored using the xCELLigence system. NPC, Nasopharyngeal carcinoma; DMSO, dimethyl sulfoxide.

compared with that of silvestrol, indicating increased efficacy and suggesting that stereoisomerism may be involved. DMSO showed no influence on cell viability (data not shown). 


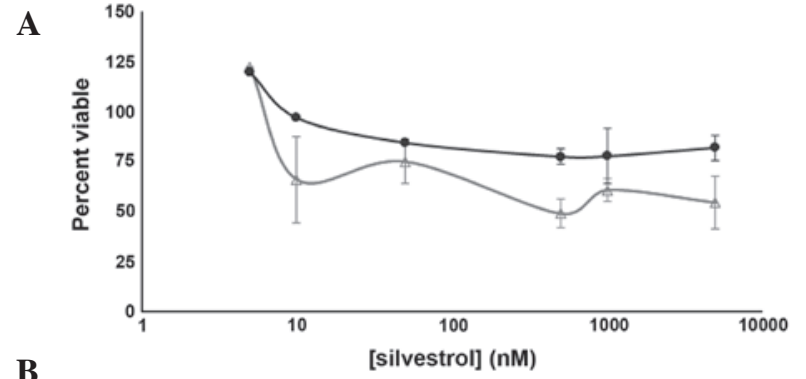

B

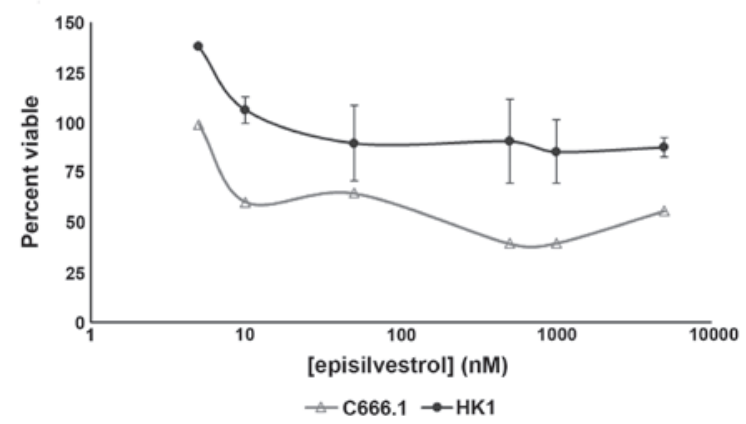

Figure 6. Viability curves of HK1 and C666.1 cells $24 \mathrm{~h}$ following culture in (A) silvestrol or (B) episilvestrol, as determined by the MTS assay. Two independent experiments were conducted. The results are presented as the mean \pm standard deviation.

Silvestrol and episilvestrol do not induce apoptosis. To determine if inhibited cell proliferation was associated with apoptosis induction, HK1 and C666.1 cells cultured in silvestrol or episilvestrol were subjected to Annexin V-FITC and propidium iodide assay, and apoptotic cells were identified by flow cytometry. Following $24 \mathrm{~h}$ exposure to $50 \mathrm{nM}$ silvestrol or episilvestrol, there was no significant difference in the percentage of apoptotic cells between the treated cells and the control (Fig. 7A). Therefore, apoptosis did not account for the differences in cell proliferation observed in the present study. Cisplatin, an NPC chemotherapy drug, was used for instrument set-up and comparison of apoptosis induction (data not shown). Consistent with the flow cytometry results, no differences were detected in silvestrol or episilvestrol-treated cells compared with the control, as determined by the IN Cell 2000 high content cell analyzer. However, apoptosis was detected in cisplatin-treated HK1 and C666.1 cells, as shown in Fig. 7B and demonstrated by the greater quantity of green (Annexin V-FITC) and red (propidium iodide) stained cells. Cisplatin is used in cancer therapy due to its apoptosis-inducing activity. The amount of green and red fluorescence in silvestrol or episilvestrol-treated cells and vehicle control-treated cells were similar and were markedly lower compared with cisplatin-treated cells. These results were concordant with the flow cytometry results which demonstrated that silvestrol and episilvestrol did not induce apoptosis with the experimental dose and time.

Cell cycle analysis assay. DNA synthesis was monitored by BrdU-labelling and cell cycle progression by flow cytometry. When BrdU is added to cell cultures it is incorporated into newly synthesized DNA of cells entering and progressing through the S-phase (DNA synthesis phase) of the cell cycle. In HK1 cells, $24 \mathrm{~h}$ after cell culture in $50 \mathrm{nM}$ silvestrol or episilvestrol, there was no observable difference in BrdU-positive cells compared with the control (Fig. 8A). Silvestrol or episilvestrol did not affect cell distribution in the S-phase. However, there was a significant reduction of cells in the $\mathrm{G}_{0} / \mathrm{G}_{1}$ phase (silvestrol, $\mathrm{P}=0.011$; episilvestrol, $\mathrm{P}=0.002$ ) and increase in the $\mathrm{G}_{2} / \mathrm{M}$ phase (silvestrol, $\mathrm{P}=0.006$; episilvestrol, $\mathrm{P}=0.016$ ) in treated cells compared with the control (Fig. 8B). This cell cycle inhibition observed in HK1 cells corresponds to the plateaued cell index observed following xCELLigence assay. The cells were neither proliferating nor dying. There was no significant observable effect on C666.1 cells within $24 \mathrm{~h}$. However, after $48 \mathrm{~h}$ there was a significant reduction in BrdU-positive C666.1 cells (silvestrol, $\mathrm{P}=0.019$; episilvestrol, $\mathrm{P}=0.042$ ) compared with the control (Fig. 9A). This was accompanied by a significant increase in $\mathrm{G}_{2} / \mathrm{M}$ phase cells (silvestrol, $\mathrm{P}=0.000$; episilvestrol, $\mathrm{P}=0.000$ ) compared with the control (Fig. 9B). The reduction of DNA synthesis in C666.1 cells may have caused the cell index (determined by the xCELLigence assay) to rapidly decrease. These differences in cellular effects and the time point at which the compounds exert their effects support the hypothesis that the activity of silvestrol and episilvestrol is cell-specific in HK1 and C666.1 cells.

Silvestrol and episilvestrol display synergistic effects in combination with cisplatin. As the results of the $\mathrm{xCEL}-$ Ligence system and the MTS cell viability assay revealed that silvestrol or episilvestrol alone did not exert significant effects on HK1, both compounds were further investigated to determine whether they were able to sensitize NPC cells to the treatment and effect of cisplatin. Using CalcuSyn software, the CI was determined to ascertain the combinatorial effect of silvestrol or episilvestrol with cisplatin. The CI values are presented in Table I. The CI method (14), revealed a remarkable synergistic activity in HK1 cells treated simultaneously with silvestrol or episilvestrol and cisplatin (Fig. 10). Similarly, in C666.1 cells, silvestrol or episilvestrol synergized with cisplatin.

\section{Discussion}

Plants have been an important source of novel drugs of natural origin over the past decade (5). Our previous study demonstrated that quercetin, a polyphenolic flavonoid found in vegetables and fruits, and trans-cinnamaldehyde obtained from the stem bark of Cinnamomum burmannii, exhibited synergism with cisplatin in inhibiting the growth of NPC cells $(15,16)$. Notably, the skeletal structures of the rocaglamide derivatives include a flavonoid unit and a cinnamic acid amide moiety (9). The synthesis of racemic rocaglamide from the benzofuran intermediate with trans-cinnamaldehyde was described by Pan et al (9).

The results of the present study showed that silvestrol and episilvestrol were able to inhibit the proliferation of EBV-positive C666.1 and EBV-negative HK1 NPC cells. NPC is often associated with the EBV. EBV-infected NPC cells exhibit type II latency and may express among others, latent membrane proteins 1 and 2 (LMP12A and B), of which LMP1 is oncogenic (3). According to Patton et al (17), silvestrol modulates direct anti-tumor activity against EBV-associated lymphomas while sparing innate and antigen-specific adaptive 
A

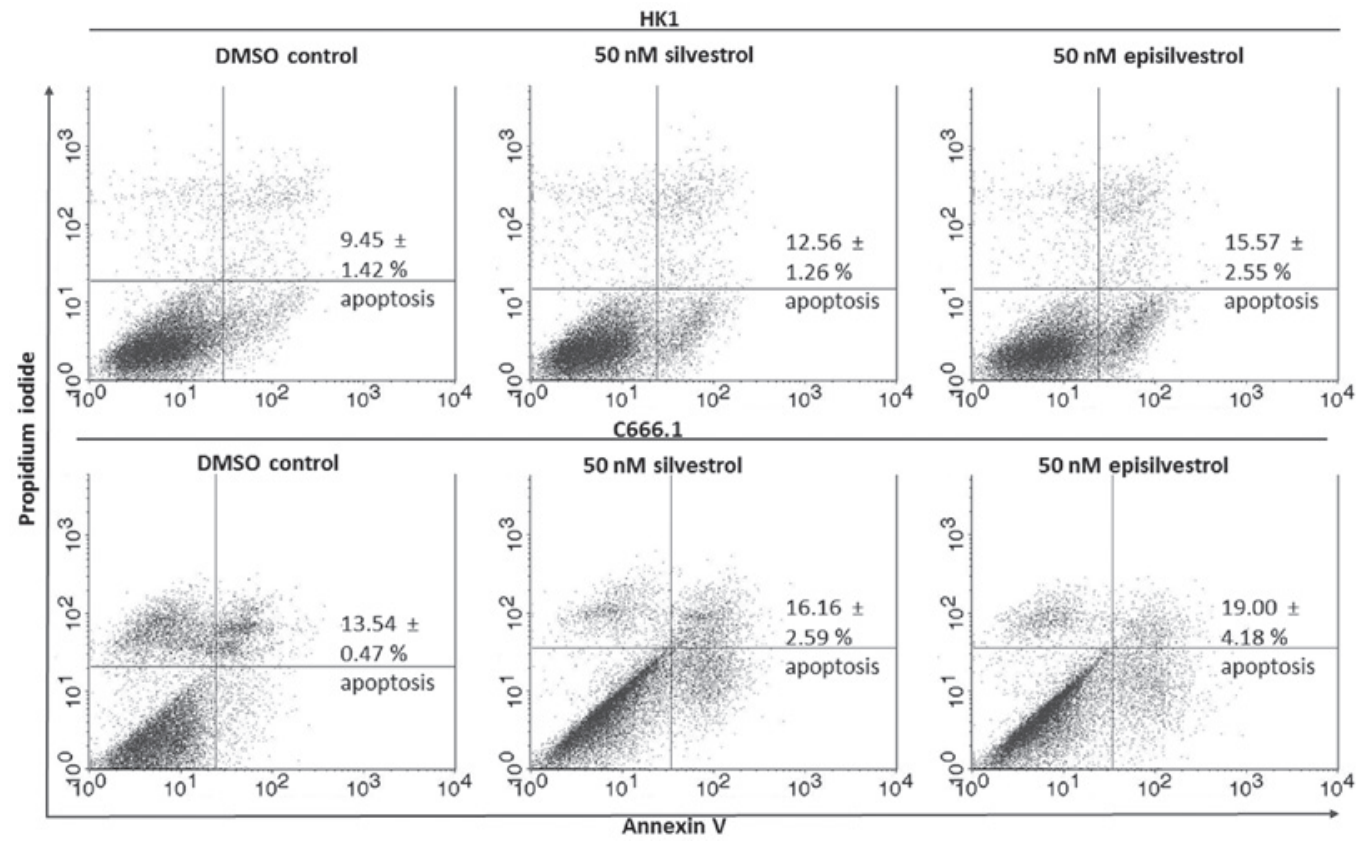

B

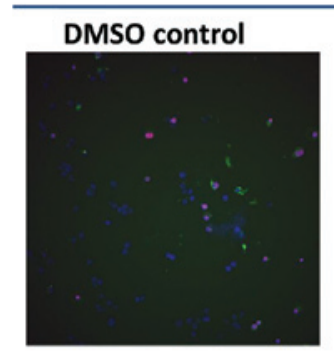

HK1

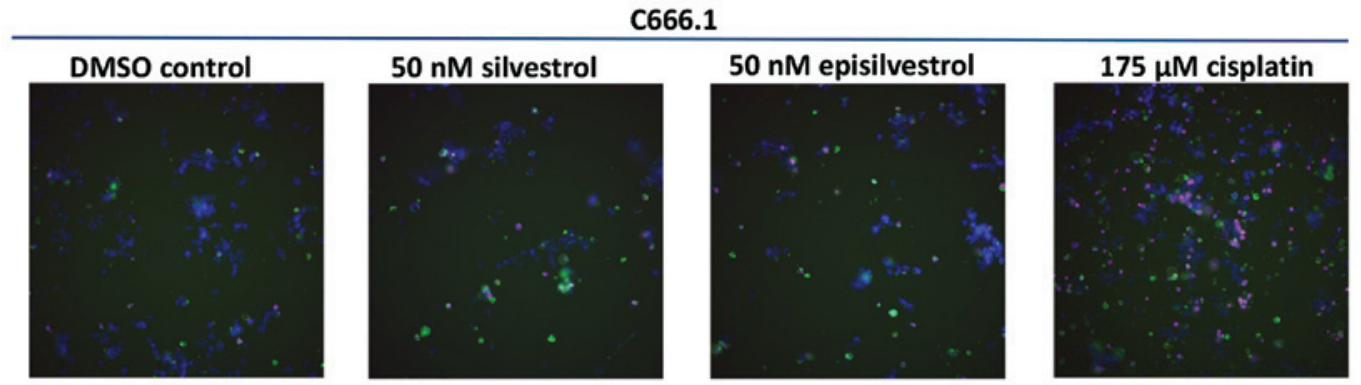

Figure 7. (A) Flow cytometry apoptosis assay with Annexin V-FITC and propidium iodide dual staining of HK1 and C666.1 cells exposed to $50 \mathrm{nM}$ silvestrol or episilvestrol $24 \mathrm{~h}$ prior to analysis. The average percentage \pm standard deviation of total apoptotic cells is shown. (B) High content analysis for apoptosis. Representative fused Hoechst 33342, Annexin V-FITC and propidium iodide fluorescence images of cells $24 \mathrm{~h}$ post-treatment. A 20x objective was used in the IN Cell Analyzer 2000. FITC, fluorescein isothiocyanate; DMSO, dimethyl sulfoxide.

immunity. There is an urgent requirement for the development of anti-cancer agents that are effective against EBV-positive diseases. The study on EBV-transformed lymphoblastoid cell lines (EBV-LCLs) demonstrated that silvestrol (2-50 nM) was highly potent against anti-proliferation, with minimal cell death (18). These results were concordant with those of the present study that demonstrated that the inhibition of NPC cell proliferation was not accompanied by apoptosis induction at $50 \mathrm{nM}$. Notably, the study demonstrated that the anti-proliferative activity of silvestrol was associated with the loss of LMP1 expression (18). In addition, it has previously been reported that silvestrol treatment had indirect anti-proliferative effects on EBV-transformed lymphoblastoid cell lines by enhancing the innate immune function (17).
As a single agent, silvestrol was markedly effective, with $\mathrm{IC}_{50}$ values of 4-10 $\mathrm{nM}$. Silvestrol exhibits nanomolar potency against multiple solid tumour cell lines $(10,19,20)$. Similarly, the data of the present study obtained from the preliminary screening experiments conducted on NCI-H460 and MCF-7 showed a similar potency. The NPC results were in concordance with these findings. However, contrary to a previous study (10) which stated that silvestrol was approximately three times more potent than episilvestrol, the results herein suggested that episilvestrol had higher efficacy against NPC cells.

The present flow cytometry data indicated that silvestrol and episilvestrol inhibit the $\mathrm{G}_{2} / \mathrm{M}$ transition in the NPC cell cycle. A previous study evaluating the cytotoxicity of silvestrol in LNCaP human prostate carcinoma cells indicated a similar 
A

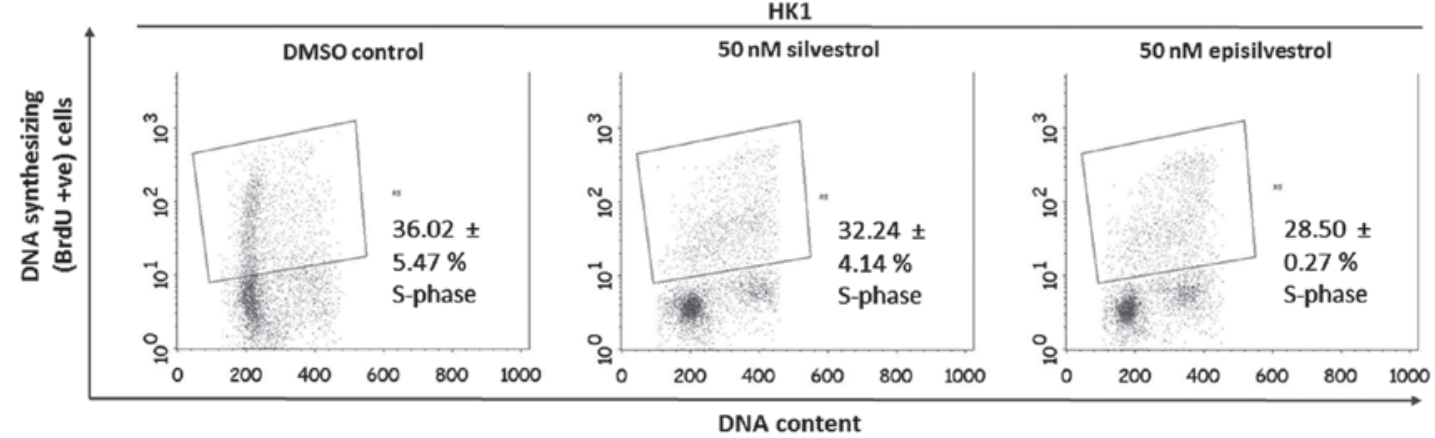

B

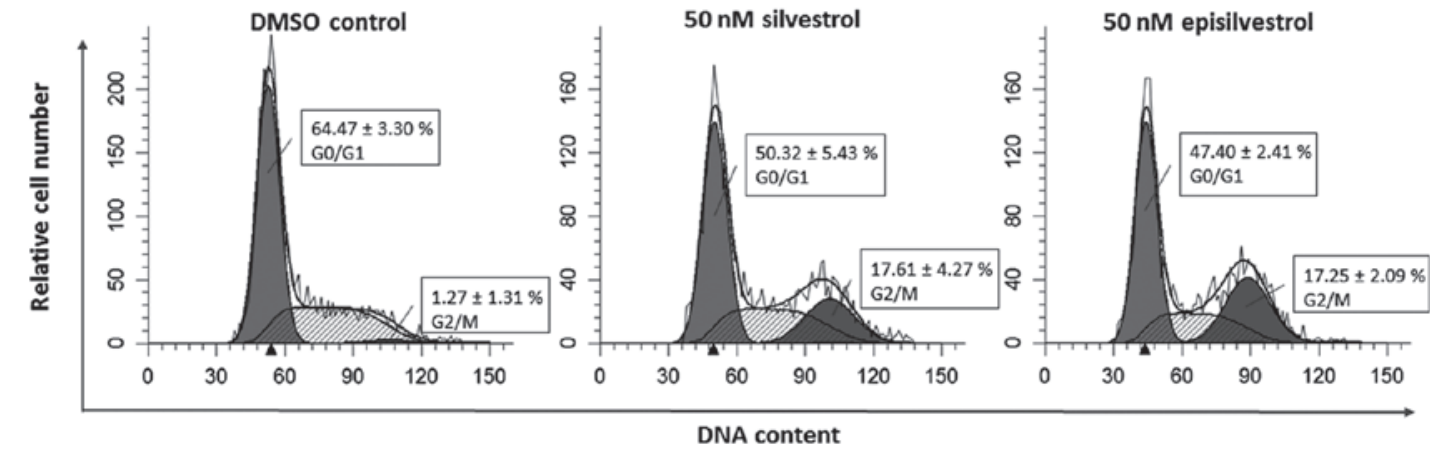

Figure 8. (A) DNA synthesis flow cytometry of HK1 cells that incorporated BrdU after $24 \mathrm{~h}$ (rectangle gates). (B) Cell cycle progression analysis by software modelling. The average percentage \pm standard deviation of total cells in the various phases are shown. DMSO, dimethyl sulfoxide; BrdU, bromodeoxyuridine.

A

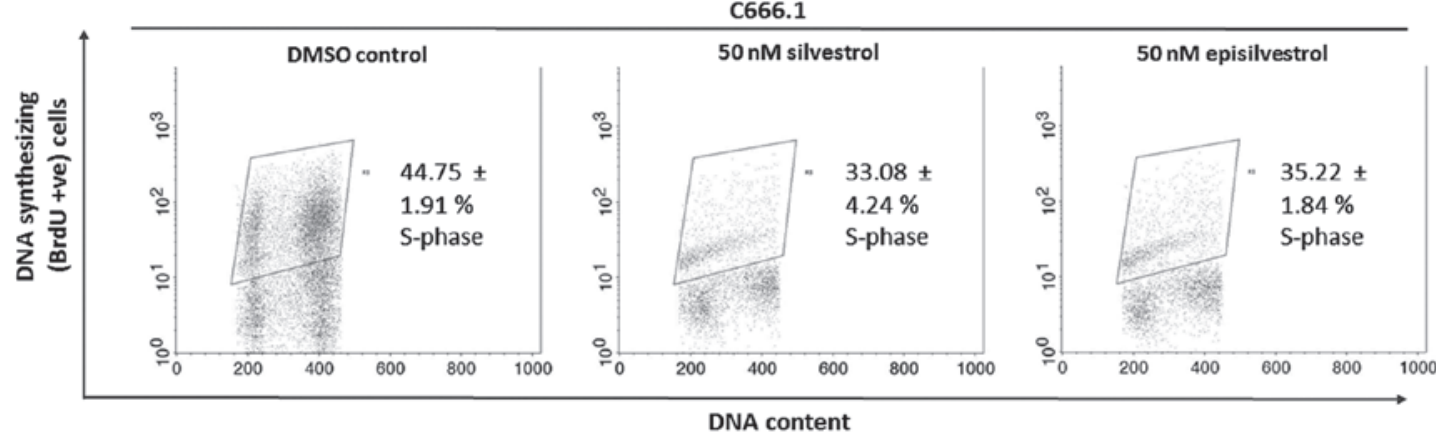

B
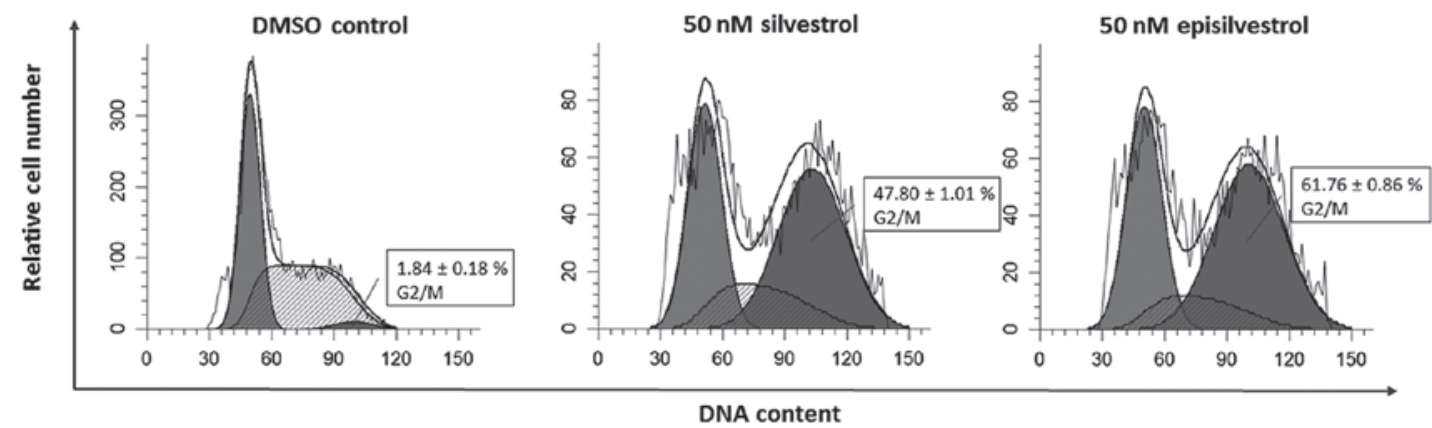

Figure 9. (A) DNA synthesis flow cytometry of C666.1 cells that incorporated BrdU after $48 \mathrm{~h}$ (rectangle gates). (B) Cell cycle progression analysis by software modelling. Mean percentage \pm standard deviation of total cells in the various phases are shown. DMSO, dimethyl sulfoxide; BrdU, bromodeoxyuridine.

mode of action (21). In addition, another study demonstrated that rocaglaol, which is a cyclopenta[b]benzofuran flavagline, inhibited $\mathrm{G}_{2} / \mathrm{M}$ cell cycle progression (22). Tumours often have increased proliferation rates. Evaluation of the percentage of cells in $\mathrm{S}$ phase may be useful, as it may convey prognostic value. To evaluate the number of cells in $\mathrm{S}$ phase, a pulse-chase experiment was performed herein. In C666.1 cells, silvestrol and episilvestrol were able to reduce DNA synthesis. However, this was not observed in HK1 cells under the present experimental conditions. Conversely, Kim et al (19) reported that while certain members of the plant-derived cyclopenta[b] benzofuran class were cytostatic against various human cancer cell lines, silvestrol exhibited a cytotoxic rather than cytostatic effect, as determined by a colony formation assay with LNCaP 

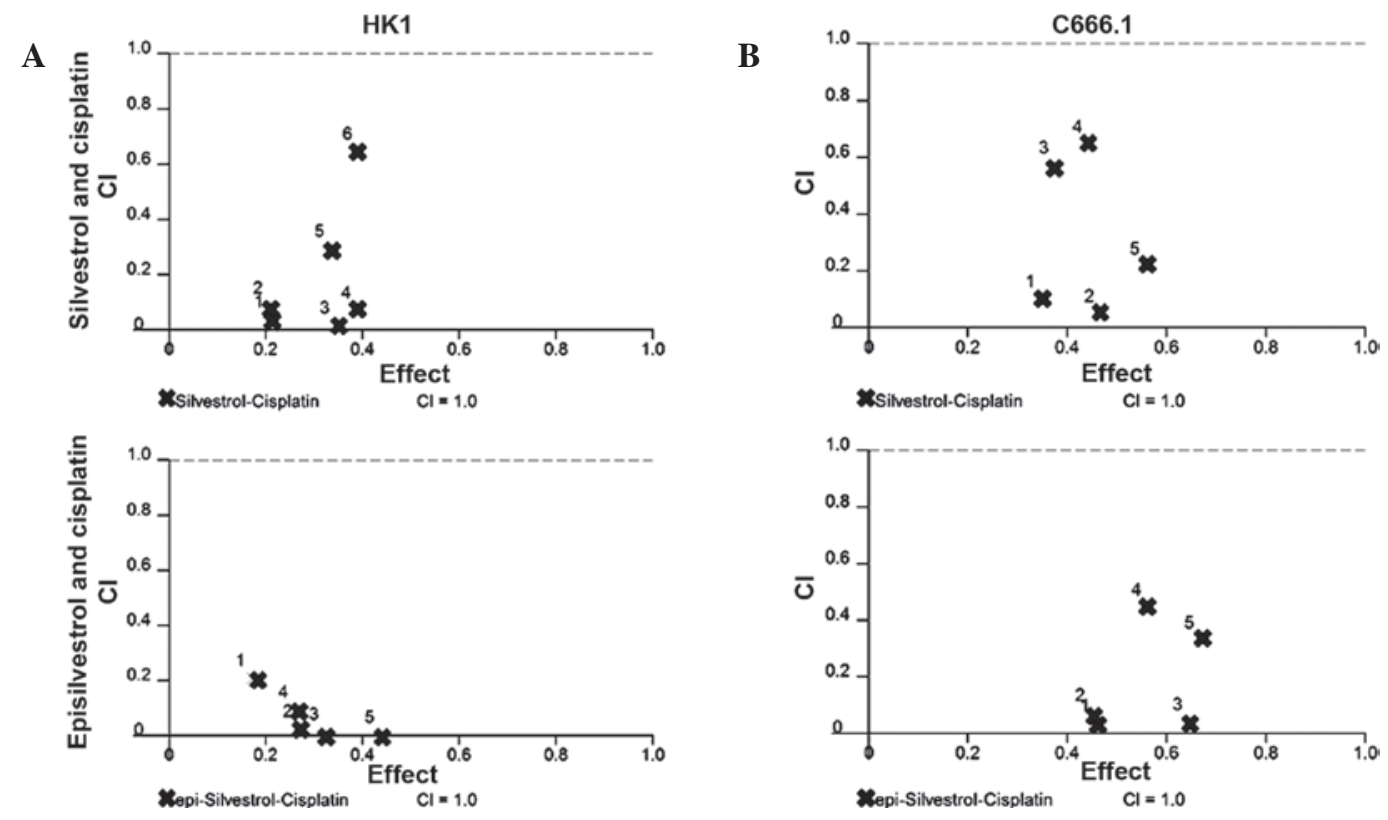

Figure 10. $\mathrm{F}_{\mathrm{a}}$-CI plots displaying the synergistic cytotoxic effect of $24 \mathrm{~h}$ simultaneous exposure to silvestrol or episilvestrol with cisplatin on (A) HK1 and (B) C666.1 cells. Values below the dashed line imply synergism. CI=1 is additive; $\mathrm{CI}>1$ indicates antagonism. CI, combination index; $\mathrm{F}_{\mathrm{a}}$, fraction affected.

cells. Furthermore, silvestrol induced an apoptotic response. A previous study demonstrated that silvestrol induced cell growth arrest and apoptosis in AML cell lines and primary blasts (23). Another study reported the ability of silvestrol to inhibit eIF4F-dependent translation correlated with the ability to inhibit cell proliferation (24).

Concurrent chemotherapy and radiotherapy or chemoradiation is the standard treatment regimen for NPC. The method of treatment uses the chemotherapy drug cisplatin, 5 -fluorouracil, or a combination of both in addition to radiotherapy to achieve local control of the disease (4). However, treatment of metastatic NPC remains a clinical challenge. One of the strategies currently under investigation involves the use of additional therapeutic agents in combination to the standard chemoradiation regimen to treat patients with metastatic NPC. The results of the present study demonstrated that silvestrol and episilvestrol, natural products from the A. stellatopilosa tree, exhibit in vitro synergism with cisplatin for the inhibition of NPC cell growth. Previous studies have shown that tumour cells can be sensitized by silvestrol to standard chemotherapeutic agents such as doxorubicin $(8,25)$ or daunorubicin, etoposide or cytarabinose-C (26), thereby producing improved therapeutic effects. One recent study demonstrated that the growth of hepatocellular cancer cell lines was inhibited by silvestrol, and that there was a synergistic effect of the compound with chemotherapy drugs sorafenib and rapamycin (27). The data obtained from the present investigation demonstrated that concomitant treatment of silvestrol or episilvestrol with cisplatin at various ratios showed marked synergistic growth inhibitory effects on NPC cells. The results also showed that the dose reduction index, which indicates how many fold the dose of each drug may be reduced in a synergistic combination compared with the dose of that drug alone, were $>1$. This is advantageous as a reduced dose leads to decreased toxicity in the host, while maintaining the same therapeutic efficacy (14). When administered as a single agent to nude mice, in vivo anti-tumour effects of silvestrol were observed with no obvious toxicity (8). This allowed the use of silvestrol with established agents as a novel therapeutic strategy $(9,26)$.

Silvestrol has been shown to exhibit in vivo anti-tumor activity in B-cell acute lymphoblastic leukemia and chronic lymphocytic leukemia (20). In a previous in vivo study, silvestrol administration suppressed the growth of MDA-MB-231 breast cancer and PC-3 human prostate cancer xenografts (8). Notably, vehicle and silvestrol-treated animals displayed similar blood cell profiles and silvestrol appeared to be well-tolerated in animals. However, the use of silvestrol in studies in vivo is hindered by poor absorption, distribution, metabolism and secretion and sensitivity to P-glycoprotein-mediated multidrug resistance (22). Gupta et al (28) described silvestrol as a substrate of phosphoglycolate phosphatase that was likely to result in the poor oral absorption of silvestrol observed in mice. Phosphoglycolate phosphatase efflux is a crucial aspect to overcome for the successful drug development of oral formulations (28). It was reported that intraperitoneal systemic availability was $100 \%$; however, the oral bioavailability of silvestrol was markedly lower (29). A study conducting pharmacokinetics analysis of silvestrol via the development and validation of a sensitive LC-MS/MS method for accurate quantification of silvestrol in mouse plasma has previously been described (29). The data suggested an overall favorable pharmacokinetic profile of silvestrol in mice (29).

In conclusion, silvestrol and episilvestrol may function as adjunct therapeutic agents in the standard NPC treatment regimen of cisplatin and 5-fluorouracil. The synergism of these compounds with standard therapeutic agents may help in reducing drug toxicity in patients with NPC. Further investigation is required in order to understand the exact mechanism underlying the synergism between silvestrol or episilvestrol and cisplatin. 


\section{Acknowledgements}

The authors thank the Director General of Health (Malaysia) Datuk Dr Noor Hisham Bin Abdullah for permission to publish this article and the Director of the Institute for Medical Research, Dr Jasbir Singh Dhaliwal for support. The present study was financially supported by the Ministry of Health of Malaysia (grant no. NMRR-14-815-22074).

\section{References}

1. Wei WI and Sham JS: Nasopharyngeal carcinoma. Lancet 365: 2041-2054, 2005.

2. Devi BC, Pisani P, Tang TS and Parkin DM: High incidence of nasopharyngeal carcinoma in native people of Sarawak, Borneo island. Cancer Epidemiol Biomarkers Prev 13: 482-486, 2004.

3. Tao Q and Chang AT: Nasopharyngeal carcinoma: Molecular pathogenesis and therapeutic developments. Expert Rev Mol Med 9: 1-24, 2007.

4. Wee J: Nasopharyngeal cancer: A promising future. Lancet Oncolo 13: 116-118, 2012.

5. Kinghorn AD, Pan L, Fletcher JN and Chai H: The relevance of higher plants in lead compound discovery programs. J Nat Prod 74: 1539-1555, 2011.

6. Pannel CM: Aglaia (Meliaceae). In: Tree flora of Sabah and Sarawak. Vol 6. Soepadmo E, Saw LG, Chung RCK and Kiew R (eds). Forest Research Institute Malaysia, Kuala Lumpur, pp27-107, 2007.

7. Ng BL, Omarzuki M, Lau GS, Pannel CM and Yeo TC: A nucleotide signature for indentification of Aglaia stellatopilosa pannel. Mol Biotechnol 56: 671-679, 2014.

8. Cencic R, Carrier M, Galicia-Vázques G, Bordeleau ME, Sukarieh R, Bourdeau A, Brem B, Teodoro JG, Greger H, Tremblay ML, et al: Antitumor activity and mechanism of action of the cyclopenta[b]benzofuran, silvestrol. PLoS One 4: e5223, 2009.

9. Pan L, Woodard JL, Lucas DM, Fuchs JR and Kinghorn AD: Rocaglamide, silvestrol and structurally related bioactive compounds from aglaia species. Nat Prod Rep 31: 924-939, 2014.

10. Hwang BY, Su BN, Chai H, Mi Q, Kardono LB, Afriastini JJ, Riswan S, Santarsiero BD, Mesecar AD, Wild R, et al: Silvestrol and episilvestrol, potential anticancer rocaglate derivatives from Aglaia silvestris. J Org Chem 69: 3350-3358, 2004.

11. Huang DP, Ho JH, Poon YF, Chew EC, Saw D, Lui M, Li CL, Mak LS, Lai SH and Lau WH: Establishment of a cell line (NPC/HK1) from a differentiated squamous carcinoma of the nasopharynx. Int J Cancer 26: 127-132, 1980.

12. Cheung ST, Huang DP, Hui AB, Lo KW, Ko CW, Tsang YS, Wong N, Whitney BM and Lee JC: Nasopharyngeal carcinoma cell line (C666-1) consistently harbouring Epstein-Barr virus. Int J Cancer 83: 121-126, 1999.

13. Chan SY, Choy KW, Tsao SW, Tao Q, Tang T, Chung GT and Lo KW: Authentication of nasopharyngeal carcinoma tumor lines. Int J Cancer 122: 2169-2171, 2008.

14. Chou TC: Theoretical basis, experimental design, and computerized simulation of synergism and antagonism in drug combination studies. Pharmacol Rev 58: 621-681, 2006.

15. Daker M, Ahmad M and Khoo AS: Quercetin-induced inhibition and synergistic activity with cisplatin - a chemotherapeutic strategy for nasopharyngeal carcinoma cells. Cancer Cell Int 12: $34,2012$.
16. Daker M, Voon YL, Akowuah GA, Yam MF and Mariam A: Inhibitory effects of Cinnamomum burmannii Blume stem bark extract and trans-cinnamaldehyde on nasopharyngeal carcinoma cells; synergism with cisplatin. Exp Ther Med 5: 1701-1709, 2013.

17. Patton JT, Lustberg ME, Lozanski G, Garman SL, Towns WH, Drohan CM, Lehman A, Zhang X, Bolon B, Pan L, et al: The translation inhibitor silvestrol exhibits direct anti-tumor activity while preserving innate and adaptive immunity against EBV-driven lymphoproliferative disease. Oncotarget 6: 2693-2708, 2015.

18. Patton JT, Lustberg ME, Garman SL, Kinghorn AD, Pan L, Lucas DM, Grever MR and Baiocchi RA: Silvestrol Modulates Direct Anti-Tumor Activity Against Epstein-Barr Virus (EBV)-Associated Lymphomas While Sparing Innate and Antigen Specific Adaptive Immunity. Presented at 53rd ASH Annual Meeting and Exposition. (abstract 104), 2011.

19. Kim S, Hwang BY, Su BN, Chai H, Mi Q, Kinghorn AD, Wild R and Swanson SM: Silvestrol, a potential anticancer rocaglate derivative from Aglaia foveolata, induces apoptosis in $\mathrm{LNCaP}$ cells through the mitochondrial/apoptosome pathway without activation of executioner caspase-3 or -7 . Anticancer Res 27: 2175-2183, 2007.

20. Lucas DM, Edwards RB, Lozanski G, West DA, Shin JD, Vargo MA, Davis ME, Rozewski DM, Johnson AJ, Su BN, et al: The novel plant-derived agent silvestrol has B-cell selective activity in chronic lymphocytic leukemia and acute lymphoblastic leukemia in vitro and in vivo. Blood 113: 4656-4666, 2009.

21. Mi Q, Kim S, Hwang BY, Su BN, Chai H, Arbieva ZH, Kinghorn AD and Swanson SM: Silvestrol regulates G2/M checkpoint genes independent of p53 activity. Anticancer Res 26: 3349-3356, 2006.

22. Mi Q, Su BN, Chai H, Cordell GA, Farnsworth NR, Kinghorn AD and Swanson SM: Rocaglaol induces apoptosis and cell cycle arrest in LNCaP cells. Anticancer Res 26: 947-952, 2006.

23. Alachkar H, Santhanam R, Harb JG, Lucas DM, Oaks JJ, Hickey CJ, Pan L, Kinghorn AD, Caligiuri MA, Perrotti D, et al: Silvestrol exhibits significant in vivo and in vitro antileukemic activities and inhibits FLT3 and miR-155 expressions in acute myeloid leukemia. J Hematol Oncol 6: 21, 2013.

24. Boussemart L, Malka-Mahieu H, Girault I, Allard D, Hemmingsson O, Tomasic G, Thomas M, Basmadjian C, Ribeiro N, Thuaud $\mathrm{F}$, et al: eIF4F is a nexus of resistance to anti-BRAF and anti-MEK cancer therapies. Nature 513: 105-109, 2014.

25. Bordeleau ME, Robert F, Gerard B, Lindqvist L, Chen SM, Wendel HG, Brem B, Greger H, Lowe SW, Porco JA Jr and Pelletier J: Therapeutic suppression of translation initiation modulates chemosensitivity in a mouse lymphoma model. J Clin Invest 118: 2651-2660, 2008.

26. Cencic R, Carrier M, Trnkus A, Porco JA Jr, Minden M and Pelletier J: Synergistic effect of inhibiting translation initiation in combination with cytotoxic agents in acute myelogenous leukemia cells. Leuk Res 34: 535-541, 2010.

27. Kogure T, Kinghorn AD, Yan I, Bolon B, Lucas DM, Grever MR and Patel T: Therapeutic potential of the translation inhibitor silvestrol in hepatocellular cancer. PLoS One 8: e76136, 2013.

28. Gupta SV, Sass EJ, Davis ME, Edwards RB, Lozanski G, Heerema NA, Lehman A, Zhang X, Jarjoura D, Byrd JC, et al: Resistance to the translation initiation inhibitor silvestrol is mediated by ABCB1/P-glycoprotein overexpression in acute lymphoblastic leukemia cells. AAPS J 13: 357-364, 2011.

29. Saradhi UV, Gupta SV, Chiu M, Wang J, Ling Y, Liu Z, Newman DJ, Covey JM, Kinghorn AD, Marcucci G, et al: Characterization of silvestrol pharmacokinetics in mice using liquid chromatography-tandem mass spectrometry. AAPS J 13: 347-356, 2011. 